\title{
Application and development trend of unmanned aerial vehicle navigation technology
}

\author{
Haohan Bei ${ }^{1,2, *}$,Yubo Liu ${ }^{1,2}$, Wenhao $\mathrm{Li}^{1,2}$, and Ying Huang ${ }^{1,2}$ \\ ${ }^{1}$ Graduate Group, Engineering University of PAP, Xi 'an 710086, China \\ ${ }^{2}$ College of Information Engineering, Engineering University of PAP, Xi 'an 710086, China
}

Keywords: UAV, Navigation technology, Application, Development.

\begin{abstract}
UAV (Unmanned Aerial Vehicle) equipment is novel because of its remote automatic control characteristics, so navigation technology is particularly critical. It is necessary to study and apply UAV technology. This paper first analyzes the UAV navigation technology, then the different types of UAV navigation system, and finally looks forward to the development direction of UAV navigation system, hoping that through this study, we can have a general understanding of the application and development trend of UAV navigation technology, and hope that the relevant research can be further enriched and improved to guide practical application.
\end{abstract}

\section{Introduction}

UAV navigation technology plays an important role in its flight control. At present, there are many navigation technologies used by UAVs, such as satellite navigation, inertial navigation, Doppler navigation, etc. These technologies have their own characteristics. It is necessary to use reasonable navigation technology for UAV Based on specific purpose. Domestic UAV has made great progress in these years. With the advantages of information technology, UAV technology is promoted to develop continuously, and its navigation technology is upgraded accordingly.

\section{UAV navigation technology}

\subsection{UAV satellite navigation technology}

Satellite navigation system consists of user receiving equipment, global monitoring station and control station, satellite. Take GPS as an example, the navigation satellite continuously sends navigation messages. The user calculates the pseudo range (PR) according to the time consumed by the satellite signal transmission and the position of the satellite in this period of time. As a satellite navigation system, it allows the electronic receiver to determine its

Corresponding author: 547651730@qq.com 
position including longitude, latitude and time. It has the characteristics of all-weather and global, accurate navigation, no error due to time accumulation, and good real-time performance.

\subsection{UAV inertial navigation technology}

Inertial navigation system is very independent. It does not rely on external information, and does not radiate energy outward. Based on the specific initial motion conditions, through the use of inertial sensors and with the reference of inertial space, the linear motion and angular motion parameters of the inertial navigation system are measured. The accuracy of inertial navigation system increases with the increase of the accuracy of each sensor, and the actual space position will drift and increase with the accumulation of time. Combined with the difference of environment and performance, different kinds of inertial navigation systems can be widely used on UAV. Take a strap down inertial navigation system as an example, in this formula, fn and fb represent the system specific forces of inertial coordinates and carrier coordinates respectively; $R_{M}$ and $R_{N}$ represent the radius of meridian curvature and prime unitary curvature respectively [1]. The following formula is used to calculate the strapdown inertial navigation position:

$$
\begin{gathered}
f^{n}=T f^{b} \\
\left.\dot{V}_{\text {en }}=f+2 \omega_{i e}+\omega_{e n}\right) * V_{\text {en }}+g \\
\dot{\varphi}=\frac{V_{\text {eny }}^{n}}{R_{M}} \\
\dot{\lambda}=\frac{V_{\text {enx }}^{n}}{R_{N}} \sec \varphi \\
\dot{h}=V_{e n z}^{n}
\end{gathered}
$$

\subsection{UAV radio tracking technology}

In general, the radio tracking and measuring system consists of the equipment on the aircraft and the ground. Its working mechanism is to modulate the relevant signal on the radio carrier. The radio carrier is generated by the transmitter and radiated to space in the form of an antenna, which is received and transmitted by the transponder on the aircraft. Of course, it is also feasible to transmit it directly to the ground; or the radio signal is sent to the ground by the beacon on the aircraft. Some short-range UAVs have a short flight distance of only $80 \mathrm{~km}$ to $100 \mathrm{~km}$. Among them, it can be used for battlefield reconnaissance and ground attack in mountainous areas, and also can be short-range coastal defense such as coastal investigation.

\subsection{UAV doppler navigation technology}

Doppler navigation is often used in aircraft. It uses the principle of Doppler effect. The 
ground speed and drift angle data will be displayed in the Doppler radar of the system. The application of navigation computer can detect the flight path of UAV. This kind of navigation not only has good autonomy, but also can respond in a short time. It can resist external interference with minimal error. It can be applied to different climate and terrain conditions. However, electromagnetic waves will inevitably be emitted during the work, and the concealment is not good.

\subsection{UAV geomagnetic navigation technology}

According to the difference of geomagnetic data processing methods, geomagnetic navigation includes two kinds: geomagnetic matching and geomagnetic filtering. At present, the former is more used in navigation application research. Firstly, the geomagnetic field characteristic quantity of a specific point in a specific section is planned effectively, and then the geomagnetic matching map is drawn into a reference map, which is saved by the carrier computer. The navigation system has excellent anti-jamming and concealment performance, is not limited by time and space, consumes low energy and has high accuracy. It can be used in cross sea guidance. However, it needs to save huge geomagnetic data, and its real-time performance is greatly restricted by the computer data processing ability.

\subsection{UAV integrated navigation technology}

Single navigation has its advantages and disadvantages. In order to form a complementary, integrated navigation has emerged.

Inertial GPS navigation. The inertial system corrects the information through the application of sensors, which makes the aerial alignment become a reality and ensures the stability of UAV in flight. The application of inertial system can obtain high GPS tracking and positioning accuracy, make the receiver obtain outstanding anti-interference and dynamic performance, get the effect of GPS integrity detection, and reduce the synchronization error to a low level.

Inertial terrain matching navigation. The terrain matching error is very low, and its application can solve the problem of cumulative error of inertial navigation, so as to achieve higher navigation and positioning accuracy. Because the terrain matching system has the advantages of small error and autonomy, the land information can be obtained continuously by accessing the multi-image sensing system.

Inertial Doppler navigation. Inertial Doppler navigation effectively makes up for the problem that Doppler navigation is affected by terrain factors, and improves the accuracy of inertial navigation, which makes UAV obtain excellent concealment [2].

Inertial geomagnetic navigation. Inertial navigation has the problem of accumulated error, and geomagnetic matching technology can solve this problem well. Inertial navigation has the characteristics of short-term high-precision, which helps to improve the geomagnetic matching interference, and then makes the navigation system obtain excellent concealment and applicability, which is the key issue to be discussed in the field of UAV navigation at this stage [3].

GPS-dead reckoning navigation. Based on GPS failure, the air speed, wind speed, wind direction and heading are calculated by using atmospheric data, and the ground speed and track angle are finally obtained [4]. When the GPS signal is very good, the track calculation data can correct the error of GPS positioning information, obtain higher navigation system accuracy, ensure the safe operation of UAV, improve the survey level, and avoid relying too much on measurement and control station and radar [5]. 


\section{Various navigation system of UAV}

\subsection{High altitude long endurance UAV navigation system}

High altitude and long endurance UAV undertake the task of long-range reconnaissance and surveillance. The altitude of UAV is more than $1.5 \mathrm{~km}$, and its endurance time is more than 24h. At the same time, it begins to have attack capability. Global Hawk UAV is very typical, its navigation system is very stable and high precision. This kind of UAV carries out reconnaissance at the altitude of about $20 \mathrm{~km}$, and the existence of the operating environment requires the navigation system to have special performance. The hardware and software of Global Hawk UAV navigation system adopt double redundancy design, fault detection and isolation algorithm of embedded software, information fusion algorithm, etc. The performance of Global Hawk navigation system and RQ-4A Global Hawk UAV are shown in Table 1. and Fig. 1.

Table 1. Performance of "Global Hawk" navigation system.

\begin{tabular}{|c|c|c|c|c|c|}
\hline \multirow{2}{*}{ Performance } & $\begin{array}{c}\text { Wingspan } \\
(\mathrm{m})\end{array}$ & $\begin{array}{c}\text { Length } \\
(\mathrm{m})\end{array}$ & $\begin{array}{c}\text { Maximum } \\
\text { takeoff mass } \\
(\mathrm{kg})\end{array}$ & $\begin{array}{c}\text { Maximum } \\
\text { endurance time } \\
(\mathrm{h})\end{array}$ & $\begin{array}{c}\text { Maximum } \\
\text { flight } \\
\text { altitude(m) }\end{array}$ \\
\cline { 2 - 6 } & 39.9 & 14.5 & 14628 & 35 & 19800 \\
\hline
\end{tabular}

Fig. 1. "RQ-4A Global Hawk" UAV.

\subsection{Medium range or Tactical UAV navigation system}

At present, unmanned combat aircraft is often used to suppress the enemy's air defense system and attack the key maneuvering targets on the ground. Based on the mission characteristics of reconnaissance UAV, satellite navigation/inertial navigation is often used in the navigation system. Attacking UAV and UAV are often subject to electromagnetic interference when they are in combat environment with the enemy. In order to hide itself, it is generally at a low flight altitude. This UAV uses inertial navigation/terrain matching navigation mode in the navigation mode. The "undead bird" medium range unmanned reconnaissance aircraft played an important role in the Kosovo war. INS is corrected by the difference of position and velocity between GPS and ins. This kind of integrated system is not complicated, and the two systems keep independence, which ensures the abundance of navigation information. the performance and speed of navigation system of "Undead Bird" navigation system is shown in Table 2. and Fig. 2. 
Table 2. Performance of "Undead Bird" navigation system.

\begin{tabular}{|c|c|c|c|c|c|}
\hline \multirow{2}{*}{$\begin{array}{c}\text { Performan } \\
\text { ce }\end{array}$} & $\begin{array}{c}\text { Wingspan } \\
(\mathrm{m})\end{array}$ & $\begin{array}{c}\text { Cruising } \\
\text { speed } \\
(\mathrm{km} / \mathrm{h})\end{array}$ & $\begin{array}{c}\text { Maximum } \\
\text { takeoff mass } \\
(\mathrm{kg})\end{array}$ & $\begin{array}{c}\text { Maximum } \\
\text { endurance time } \\
(\mathrm{h})\end{array}$ & $\begin{array}{c}\text { Mission } \\
\text { radius }(\mathrm{km})\end{array}$ \\
\cline { 2 - 6 } & 5.5 & 158 & 177 & 4 & 50 \\
\hline
\end{tabular}

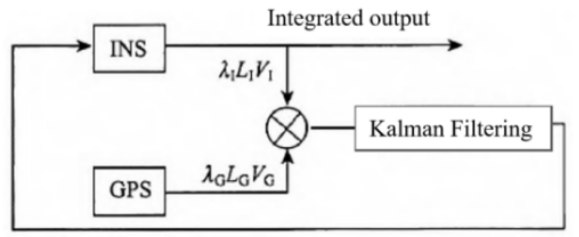

Fig. 2. Speed position synthesis of navigation system.

\section{Future development of UAV navigation technology}

\subsection{Data fusion and high-performance navigation}

Kalman filter is the key component of navigation system, which makes data processing possible. At present, China has developed a data fusion technology scheme. For example, through the use of adaptive filtering technology, the measured data are collected, the model parameters, state gain matrix, noise are dynamically analyzed, and error correction is carried out to ensure that the filtering is correct and the optimal value of the collected data is obtained. Moreover, the artificial intelligence technology is gradually improved. The artificial intelligence technology in development includes neural network and wavelet transform, which can greatly strengthen the information fusion ability of integrated navigation, dynamically analyze the flight status of UAV Based on big data, and ensure the normal flight of UAV through intelligent system, and realize unattended flight [2].

\subsection{New inertial navigation system}

By analyzing the types of integrated navigation, it is not difficult to find that many integrated navigation systems are inseparable from inertial navigation technology. The industry attaches great importance to the improvement of inertial navigation. Through the efforts of scientific and technological workers, laser inertial navigation and optical fiber inertial navigation have been developed at this stage [6]. With the maturity of information integration technology, silicon micro resonant accelerometer has been widely used, which simplifies the volume and weight of UAV navigation, reduces the energy consumption and improves the flexibility of flight. In the future, high-precision technology and integrated technology will be continuously improved, and the excellent precision inertial navigation will be used to enhance the performance of integrated navigation.

\subsection{Self adaptive and intelligent navigation capability}

In recent years, the development of UAV system has begun to trend towards multi mission system. Its types include not only micro and small UAVs, but also high-altitude long endurance UAVs, achieving the effect of full airspace coverage. It is believed that in the future, the application requirements and operational environment will be more complex and 
uncertain. Generally, the flight plan will not be followed in advance, and the flight plan will be adjusted according to the situation. In case of emergency, the navigation system is required to use the corresponding navigation technology, such as adaptive technology, neural network technology, etc., in combination with the mission characteristics and the combat environment Appropriate navigation mode [7]. In the future, the development of UAV navigation system will be uncertain about the environment, which requires certain navigation capabilities, such as self-adaptability and intelligence.

\section{Conclusion}

Through the above analysis, we understand the UAV navigation methods, each navigation method has its own characteristics, in the specific application needs to be considered. Based on the defects of single navigation mode, some data fusion technologies, such as Kalman filter, can be used to achieve the purpose of integrated navigation and obtain higher navigation accuracy. Each navigation method should consider the actual situation and select the corresponding combination mode [8]. UAV navigation technology involves a lot of interdisciplinary knowledge. How to improve UAV navigation accuracy, optimize data fusion algorithm and simplify system structure are the hot topics and directions of UAV navigation technology to continue to explore.

\section{References}

1. Zhou Jianmin, Kang Yong, Liu Wei. Application and development trend of UAV navigation technology [J]. Journal of China Academy of Electronic Sciences, 2015,10 (03): 274-277+ 286 .

2. Xu Xiaowei, Lai Jizhou, Lv Pin, et al. Research status and progress of multi UAV cooperative navigation technology [J]. Navigation positioning and timing, 2017,4 (04): $1-9$.

3. Wang Yalin, Yang Zhongsheng, Wang Tong, et al. Overview of unmanned system development in 2019 [J]. Unmanned system technology, 2019, 2 (06): 53-57.

4. Zhao Yu, Zhang Xiaoyi, Liu Guangyi. Path optimization under multi UAV cooperative positioning [J]. Signal processing, 2019,35 (04): 712-719.

5. Pan Ruihong, Xu Shenghong. Multi UAV cooperative navigation algorithm based on geometric characteristics [J]. Journal of Ordnance Equipment Engineering, 2017,38 (10): 55-59+96.

6. Yan Maode, Zhu Xu, Zhang Xunxun, et al. high precision formation control strategy for three-dimensional multiple UAVs based on consistency [J]. Frontiers of Information Technology \& Electronic Engineering, 2017,18 (07): 968-978.

7. Jin Yabo. Mutual perception and task coordination of multiple aerial robots [D]. Civil Aviation University of China, 2015.

8. Wu Wenchao, Huang Changqiang, Song Lei, et al. Route planning of multi UAV cooperative search in uncertain environment [J]. Acta ordnance, 2011,32 (11): 1337-1342. 